\title{
ESCLEROSIS MÚLTIPLE MANIFESTÁNDOSE CLINICAMENTE POR UN SINDROME MEDULAR CERVICAL DE EVOLUCIÓN LENTAMENTE PROGRESIVA A LO LARGO DE 16 AÑOS
}

L. BAHIAQUER-BORDAS

J. M. Sfgarha-Ohiol

La serie más provechosa de las lecciones médicas prácticas es la informada por el capítulo de los errores o al menos de aquellos casos cuya orientación diagnóstica no pudo precisarse. Por ello y por las características notables del presente caso, cuya naturaleza no pudo conocerse más que luego del estudio histopatológico, nos parece justificada la publicación de esta nota anátomo-clínica.

Como en otras ocasiones el caso "atípico", cuya expresión clínica dificultaba el diagnóstico nosológico, pertenecia al campo de la esclerosis múltiple. En efecto, aunque puede decirse que hoy día son prácticamente conocidas todas las posibilidades sintomatológicas que puede adoptar esta afección y aunque la cautela de los tratadistas ha atendido últimamente con justeza a atender más a las características globales del proceso que a sus detalles para dirigir la tarea diagnóstica, es lo cierto que las posibilidades anatómicas y clínicas de esta neuropatía desbordan todavía tales intentos y de cuando en cuando comprobamos casos que han escapado a nuestra visión.

Nuestra presente observación quebrantaba abiertamente lo que podemos considerar como las grandes lineas de conjunto propias del quadro clínico de la esclerosis múltiple, tal como fueron conceptuadas por nosotros en anteriores ocasiones ${ }^{1}$. Decíamos entonces que para orientar el diagnóstico clínico de esta neuropatía era menester atender a las siguientes normas globales: a) la comprobación, por razonamiento fisiopatológico de los síntomas, de la afectación concurrente de determinados sistemas electivamente interesados en ella; b) una evolución adecuada, lo más generalmente a brotes. Si bien se mira la primera de estas pautas atiende a la consideración del proceso en un momento dado, visto en una

Clínica de Neurología del Hospital de la Sta. Cruz y San Pablo (Prof. L. Barraquer Ferré); Servicio de Geriatría (Dr. M. Pañella) del Hospital Municipal de N. S. de la Esperanza; Servicios de Neurocirugía (Dr. E. Tolosa) y de Anatomía Patológica y Laboratorio (Dr. R. Roca de Viñals) del Instituto Neurológico Municipal de Barcelona. 
suerte de sección transversal, mientras que la segunda toma en consideración su despliegue temporal. Podemos decir que una considera la afección en el espacio y la otra en el tiempo.

Los mismos puntos de vista fueron expuestos más recientemente con J. Vila-Badó ${ }^{2}$ : "Insistimos tanto en estos conceptos generales, por cuanto estamos plenamente convencidos de que sólo así, con estas idéas, puede el médico llegar al diagnóstico de la esclerosis múltiple, diagnóstico al que difícilmente llegará si estudia separadamente cada una de las múltiples manifestaciones semiológicas y no llega a integrarlas en el espacio $y$ en el tiempo en un concepto global y sintético, unitario y positivo".

Este modo de ver coincide, con exactitud tan completa como difícil. mente previsible, con el expuesto en un reciente trabajo de conjunto sobre esclerosis múltiple derivado de la "National Multiple Sclerosis Society" en el que Schumacher ${ }^{3}$ considera como esenciales para el diagnóstico de este proceso los siguientes criterios: "la inequívoca evidencia, basada en signos neurológicos, de lesiones separadas, limitadas, en varias partes del sistema nervioso" y "la historia del desarrollo de los síntomas y el progreso subsiguiente de la afección", teniendo la presencia de brotes y de remisiones un máximo valor. $\mathrm{Y}$ advierte el autor que "el diagnóstico de esclerosis múltiple no puede ser hecho con seguridad si uno u otro, preferentemente ambos, de los dos criterios no és plenamente cumplido". La coincidencia extrema entre nuestro modo de ver y el dimanado de la Institución Norte-americana para el estudio de la afección en cuestión se hace aún mayor al hacer constar Schumacher, de acuerdo con nosotros y con la mayoría de autores, que la edad de aparición del cuadro clínico tiene asimismo una acentuada importancia orientadora. La esclerosis múltiple suele iniciarse a lo largo del tercer o cuarto decenio de la vidá.

Pues bien, contraviniendo todo lo apuntado, la historia que referimos a continuación és de una mujer que enfermó hacia los 42 años de un proceso que evolucionó de modo lentamente progresivo y cuya semiología parecía indicar la existencia de unas lesiones - o si prefiere, de una lesión - exclusiva o casi exclusivamente localizadas a un determinado nivel de la médula espinal. A la verificación este proceso se reveló ser una esclerosis múltiple.

Bien es verdad que todos cuantos hemos tratado recientemente de trazar normas fundamentales para el diagnóstico clínico de la esclerosis múltiple hemos cuidado de advertir que la evolución clásica a brotes puede faltar. Por esto con Vila-Badó escribíamos expresamente que "evolución adecuada" no equivalía necesariamente a evolución por brotes, pudiendo ser lentamente progresiva, con alguna irregularidad. Y Schumacher, continuando aún con las coincidencias señaladas, indica también que el diag. 
nóstico no puede eliminarse por la sola ausencia de la evolución más típica. También Wartenberg ${ }^{4}$, en reciente y breve comentario de conjunto, ha cuidado de dar cabida a la advertencia de que "la esclerosis múltiple no siempre sigue un curso ondulante, sino que puede progresar lentamente y aún puede permanecer estacionaria en un punto dado".

Por otra parte, es bien conocido el hecho de que muchas veces "placas" de esclerosis múltiple, aún considerables por sus dimensiones, no tienen traducción clínica, lo cual viene a reducir las características externas de multiplicidad - y multiplicidad electiva - de la afectación topográfica del sistema nervioso central.

Cuando ni la evolución es adecuada ni la đaseminación de las lesiones alcanza evidencia clínica, es lógico que la tarea diagnóstica sea extremadamente difícil. Y ello tanto más cuando el proceso se inicia a una edad que, sin constituir una verdadera rareza, tampoco es la más habitual. Por todo lo cual se echa de ver que todas las dificultades se dieron cita en la observación que a continuación exponemos.

Antonia C. M., nacida en 1892, natural de Sarrial (Tarragona), dedicada a las ocupaciones propias de su sexo. Padre muerto a los 40 años, cardiópata; madre muerta a los 62 con neumonía; han sido 9 hermanos (cuatro murieron en la infancia $y$ otro a los 30 años, ignorando causas); su marido murió últimamente de ulcus gástrico; tiene un hijo aparentemente sano. Desarrollo y datos fisiológicos nomales. Ha sufrido sarampión, amigdalitis, gastroenteritis y grippe en la pandcmia de 1916. Su enfermedad actual se inició aproximadamente por el año 1935 por dificultad motora en la extremidad inferior derecha, que la arrastraba por su punta al andar. Esta debilidad fué aumentando muy lentamente, sintiéndola poco después en extremidad inferior izquierda. Poco después, y desde entonces siempre, fué esta última extremidad la más afectada.

El 8 de octubre de 1936 acudió al Dispensario de la Clínica de Neurología del Hospital de la Sta. Cruz y San Pablo, quejándose de debilidad de extremidades inferiores $y$ dolores en la columna vertebral. Se constató una paraparesia espasmódica con exaltación de los reflejos profundos, clonus y signo de Babinski bilateral. Acentuados transtornos de esfinteres. Iigera atrofia muscular. La reacción de Wassermann en sangre fué negativa y el análisis del L.C.R. dió: células 0 , albúmina 0,25 por mil, Pandy y Nonne-Apelt negativos, benjuí coloidal 00001.22222 .00000 .0 , Wassermann negativa.

EI 21 de junio de 1950 ingresa en el Servicio Municipal de Geriatría, bajo el cuidado del Dr. R. Bracons. Cuando uno de nosotros levanta su historia en este Servicio, nos refiere que la atrofia muscular de manos apareció y progresó antes en la izquierda. Fractura de pierna derecha en 1948 consolidada en mala posición. Desde hace unos meses no se levanta nunca ni puede tampoco sentarse por su propio esfuerzo. Nunca dolores; niega parestesias. Insiste en el inicio insidioso de su afección y en que ésta ha seguido una evolución siempre paulatinamente progresiva; nunca brotes; nunca remisiones. No hay noción de traumatismo ni de infección alguna precediendo al inicio de su afección actual.

A la exploración encontramos una enferma en decúbito supino forzado. A duras penas, tomando apoyo con las manos en la cabecera de la cama, puede 
girarse y colocarse en decúbito lateral, mas fácilmente derecho. Wxtremidades inferiores: Paraplejía espástica con contractura en flexión moderada; actitud en semiflexión o flexión moderada y aducción extrema; retracciones musculotendinosas en región posterior de ambos muslos; atrofia de ambos cuadriceps, mayor del derecho; pies en ligero equino-varo; dedo gordo de ambos pies en moderada flexión dorsal, más acentuada en el lado izquierdo. Solo puede realizar algunos movimientos con la extremidad inferior derecha consistentes en discretos movimientos de los dedos, más discretos del pie y ligera oscilación lateral de la rodilla; con la extremidad inferior izquierda todos los movimientos son prácticamente imposibles. La actitud en moderada flexión de ambas rodillas está fijada por las retracciones musculotendinosas indicadas; dureza extrema de la masa muscular de la región posterior de ambos muslos, especialmente del izquierdo. Reflejos de cuadriceps, bruscos y monocinéticos, sin determinar movilización articular (fijaciones secundarias); reflejos de ambos tríceps surales bruscos, produciendo clonus de pie; maniobras de Gowers y de Bing cficientes. Clonus de ambos pies por la maniobra clásica, más fácil y evidente a izquierda. Signo de Babinski bilateral con triple retirada homolateral discreta y extension cruzada que, aunque moderada, es bien evidente. Signo de flexión dorsal refleja del pie (Babinsky) bilateral, más fácil y expresivo a izquierda. La maniobra de Sinkler-BechterewMarie-Foix produce en ambos lados el "fenómeno de los acortadores"; también puede determinarse éste por pellizcamiento de la piel del muslo. Sensibilidad táctil normal; hipoalgesia y termohipostesia más acentuadas en extremidad inferior derecha; sensibilidad vibratoria abolida hasta nivel de las crestas ilíacas; transtornos de la artrocinética en dedos de ambos pies. $\mathrm{Ni}$ que decir tiene que en extremidades inferiores la coordinación no pudo ser explorada por la paraplejía. Esfinteres: Micción subimperiosa, algunas veces incontinencia; raramente incontinencia fecal; estreñimiento habitual. Tronco y abdomen: reflejos cutáneos abdominales inferiores y medios abolidos, superiores inconstantes; muy débiles, esbozados, cuando aparecen. Hipoalgesia y termohipostesia en tronco, más acentuadas a derecha. Extremidades superiores: actitud indiferente a nivel de hombro, codo y muñeca; mano izquierda moderadamente excavada; los tres últimos dedos en semiflexión; atrofia tenar, hipotenar $\mathrm{y}$ de interóseos en esta mano; en la mano derecha se inicía la actitud excavada de la mano y una ligera flexión de los últimos dedos; atrofía incipiente. No se apreciaron fibrilaciones. Paresia acentuada de interóseos y denrás músculos propios de la mano izquierda; fuerza de prensión muy débil en esta mano; el resto de la motilidad denota también una paresia bien apreciable en esta misma extremidad. Discreta debilidad motora en el lado derecho, siendo especialmente apreciable en la extensión de la muñeca. Ligera hipertonía elástica en ambos lados. Reflejos profundos exaltados; pronador derecho policinético; el reflejo flexor de los dedos es acentuado en ambes lados, aún en el izquierdo, a pesar de la atrofia; la maniobra de Trömner es cficiente. Sensibilidad táctil normal. Hipoalgesia y termohipostesia, más acentuada en extremidad derecha. Defectos del artrocinetismo en dedos de mano izquierda. Prueba dedo-nariz: a derecha, discreto temblor, inconstante, en la fase final; a izquierda, lentitud, descomposición y dismetría moderadas, defectos que se corrigen al repetir la prueba. Lentitud en la prueba de marionetas, mayor a izquierda. No hay rebote en la prueba de Stewart-Holmes. No puede ponerse de manifesto, por ningún procedimiento, un franco temblor intencional. Pares craneales: Oculogiria normal; no diplopia; no nistagmus. Pupilas iguales y reaccionan bien a la luz. Niega disfagia. Voz normal; ni disartria, ni palabra escandida, ni dislalia de ningún otro tipo. Trigéminos normales. No atrofia, fibrilaciones, ni desviación de la lengua. Ligera insuficiencia motora de índole du- 
dosa del facial inferior derecho, que bien puede ser un predominio constitucional del izquierdo.

Resumiendo, el cuadro clinico de nuestra enferma estaba esencialmente definido por los siguientes rasgos: a) Afección iniciada hacia los 42 años de modo insidioso y que ha progresado de modo lento, y, al parecer, uniforme a lo largo de más de 15 años; sin brotes y sin remisiones. b) Paraplejía espasmódica, con ligero predominio izquierdo, con moderada contractura en flexión; automatismo medular. c) Atrofia muscular distal en ambas extremidades superiores, mayor a izquierda, sin fibrilaciones; la atrofia, clinicamente, parece más del tipo radicular que de asta anterior (tipo Aran-Ducinenne). d) Atrofias musculares moderadas en extremidades inferiores, de interpretación dudosa, pudiendo ser debidas a la inmovilización. e) Hiperreflexia profunda generalizada, aún en extremidades superiores, $y$ en ellas principalmente de aquellos reflejos integrados por debajo del sexto segmento cervical. f) Hipoalgesia y termohipostesia, mayores en el lado derecho, afectando a extremidades inferiores, tronco y extremidades superiores, predominando en aquellas $\mathrm{y} \sin$ un nivel definido. $\mathrm{g}$ ) Transtornos de la sensibilidad vibratoria $y$ artrocinética en ambas extremidades inferiores, tronco y de ésta última en la mano izquierda. h) Ligeros defectos de la coordinación en extremidades superiores, predominando a izquierda, de interpretación cuestionable, pudiendo explicarse quizá por el déficit motor y el ligero déficit de la sensibilidad profunda, tanto más cuanto que, como estos mismos transtornos, predominaban a izquierda. Nada cerebeloso seguro. No temblor intencional. No palabra escandida. i) Transtorno de esfincteres. j) Defectos considerables, casi abolición completa, de los reflejos cutáneos abdominales. k) No transtornos de la sensibilidad subjetiva; no parestesias; no dolores; solo algumas algias vagas vertebrales, principalmente en la región cervicodorsal. 1) Integridad de pares craneales (véase luego, sin embargo, el dictámen del neuro-oftalmólogo); no diplopía; no nistagmus; no síndrome pseudo-bulbar ni bulbar; tan solo debilidad cuestionable del facial inferior derecho.

La história y el síndrome inducían a pensar en una afección puramente medular. Debía decidirse si ésta era "intrínseca" o de tipo compresivo. La distribución de los transtornos sensitivos, de la atrofía y de ciertos datos de la paraplejía señalaban un predominio izquierdo del proceso. La tarea diagnósticodiferencial obligaba a las siguientes consideraciones:

1) La esclerosis lateral amiotrófica podía invocarse por la combinación de amiotrofía y paraplejía espasmódica; pero ni la larga evolución, ni la cuantía de los transtornos sensitivos, ni la total indemnidad bulbar, autorizaban tal diagnóstico. 2) Én contra de la esclerosis múltiple, entidad que ciertamente fué seriamente considerada en estos razonamientos diferenciales, hablaba muy fuertemente la ausencia de las dos normas globales que antes hemos señalado como muy propias de esta afección; por otra parte, concretando el aspecto topográfico y sindrónico, la ausencia de modificaciones de la palabra, de parestesias, de nistagmus, de diplopía, etc., apoyaban tal actitud negativa. 3) La siringomielia tampoco podia aceptarse por la distribución, no "suspendida", de los transtornos sensitivos, por la ausencia de toda modificación "trófica", por la intensidad de la paraplejía espasmódica que sobrepasaba de mucho lo usual. 4) Una mielitis infecciosa aguda podía descartarse por la sola anamnesis. 5) La posibilidad de una compresión medular podía ser invocada, aunque clínicamente el cuadro no fuese tampoco positivamente definido. En efecto, ni la larga duración del proceso, ni la ausencia de dolores de tipo radicular, ni la falta de un nivel sensitivo claramente definido, dejaban de impresionar en contra de tal posibilidad. También debía tenerse en cuenta además que los síntomas de extremidades su- 
periores no ofrecían una clara delimitación, considerados según alturas segmentarias, en lesiones y sublesionales. Contrariamente, existía una relativa imbricación de síntomas segmentarios y síntomas funiculares. Como que esta inibricación no era, sin embargo, muy considerable podía suponerse compatible con una causa compresiva algo prolongada en sentido vertical. Este dato, la lentitud evolutiva y la existencia de dolores vertebrales regionales, sin dolores radiculares, podía inducir a sospechar la presencia de una compresión atípicn, de progresión lenta, como p. ej., la compresión cifoescoliótica, una tumoración extradural, una compresión discal con reacción aracnoidítica extensa, un golioma intramedular de crecimiento lento, etc.

Veamos de nuevo, con estas ideas ya en la mente, los datos recogidos en la historia de la paciente. Los resumiremos asi: Hematología normal. V.S.G. normal. Wassemann $y$ complementarias negativas. Ligera tonalidad depresiva reactiva. Juicio, comprensión y memoria normales (exámen psiquiátrico llevado a cabo por el Dr. Solé-Sagarra). La exploración física de la paciente nos permitió apreciar que su cuello era excesíramente corto y que existía un cambio de dirección en la columna vertebral bastante marcado, en sentido ánteroposterior, a nivel cervical inferior. Solicitamos estudios radiográficos para considerar las posibilidades de un síndrome de Klippel-Feil, de una discopatía, de una cifoescolicsis, de una impresión basilar, etc. Llevados a cabo por los Drs. Modolell y Manchon pudo eliminarse cualquier malformación y al parecer también la discopatía. Tan solo se comprobó el acentuado cambio de dirección ya indicado, centrado hacia las vértebras sexta y séptina cervicales. Una manometría por punción lumbar se reveló igualmente livre a la conpresión yugular y a la abclominal, sin trazas de bloqueo. El análisis del liquido cefalo-raquideo extraído (Dr. Guardia) demostró: células 0,2 por $\mathrm{mm}^{3}$; albúmina $0,20 \mathrm{gr}$ por mil; Pandy y Nonne-Apelt negativos; benjui 00000.22000.00000.0; Wassermann negativo.

Ante estos hechos nada concreto podía añadirse ya que no aportaban ningún dato positivo. Sabido es, por otra parte, que la compresión cifoescoliótica (Coste, Sorrel, Sorrel-Déjerine, André-'Thomas, Chavany, Dereux, Subirana, Oller-Daurelea, Gopcevitch, Gegerly, etc.), de la cual contamos con varias observaciones personales, evoluciona generalmente sin algias radiculares y con transtornos acentuados de la sensibilidad dolorosa y de la térmica, sin que estos hechos, desde luego, puedan ser tomados en un inoportuno sentido específico.

Pensando en la posibilidad de una causa compresiva, que era además la única que podía of recer una esperanza de alivił, solieitamos el tránsito de la enferma desde el Servicio Municipal de Geriatría al de Neurocirugía.

Ingresa la enferma bajo el cuidado del Dr. Eduardo Tolosa el 18 de octubre de 1950. La exploración clínica llevada a cabo por el Dr. F. Durán no demuestra diferencias de valor con la que anteriormente consta. El exímen neurooftalmológico (Dr. Idoberas-Camino) contiene lo siguiente sobre fondos oculares: En O.D., disco papilar rosado-pálido-decolorado, pronunciándose decoloración por el sector temporal y borde nasal; los bordes del disco se delimitan; lit zona peripapilar en conjunto pierde caracteres; circulación arterial de tinte cobrizo y con pérdida de color y calibre de colaterales medianas y pequeñas; sospechas de atrofia secundaria. En O.I., disco papilar de un tono rosado-pálido sospechoso, pronunciándose la hemidecoloración temporal; los bordes se delimitan con pequeño anillo escleral; la circulación arterial presenta el mismo aspecto que la del O.D., con pérdida de color $y$ calibre, que se pronuncia en colaterales medianas $y$ pequeñas; zona peripapilar y polo posterior de la retina con pérdida de caracte- 
res; sospechas de atrofia secundaria. El lipiodol introducido por vía cisternal (Dr. Figuerola) descendió libremente hasta el fondo de saco dural.

El 2 de enero de 1951 se lleva a cabo una intervención quirúrgica por el Dr. E. 'Tolosa con finalidad exploradora. Laminectomía cervical de $\mathrm{C}_{2}$ a $\mathrm{C}_{7}$. La columna cervical r.uestra una incurvación bastante acentuada pero ninguna alteración morfológica digna de mención. Se abre la dura, notándose que la médula muestra una coloración anormalmente blanquecino-pálida sobre la que destaca una finísima vascularización. No distensión de las venas ni ningún otro signo de compresión. La exploración de los aspectos subaracnoideos mediante una sonda olivar demuestra que no existe ningún obstáculo en el conducto raquídeo ni por encima ni por debajo de la zona laminectomizada. Ligerísimas lesiones aracnoidíticas. Cierre de la dura y sutura habitual por planos.

Al segundo día del post-operatorio se constata proceso bronconeumónico, mereciendo destacarse que ello coincide con un brote epidémico prolongado de procesos gripales existente en la ciudad. A pesar del tratamiento a dosis masivas de penicilina y de los cardiotónicos empleados, fallece el 6 de enero de 1951.

Antes de pasar a exponer los datos anatómicos queremos hacer constar solamente que, apesar del evidente valor complementario que tienen, ni el dato positivo de la decoloración bitemporal papilar ni el dato negativo del tránsito libre del lipiodol, podían quizás apartarnos definitivamente de la indicación de una laminectomia exploradora, dadas las circunstancias globales del presente caso.

Necropsia - Dejando aparte los datos necrópsicos generales, pasaremos a relatar directamente el resultado del estudio histopatológico del sistema nervioso central, llevado a cabo por uno de nosotros. Dada la importancia de la sintomatología medular acometimos este estudio neuropatológico empezando por este órgano. Varios segmentos medulares tomados a distintos niveles fueron cortados, unos transversalmente $\mathrm{y}$ otros longitudinalmente. Mediante técnicas diversas (Nissl, Río Hortega, plata-etilaminada, grasas, etc.) fué posible observar las siguientes lesiones: Un proceso desmielinizante se extiende a lo largo de casi toda la médula, con diferente intensidad según el segmento que se considere. En la región cervical las lesiones son mixtas $y$ abarcan indistintamente a la sustancia blanca y a la sustancia gris. La lesión mielínica se acompaña de una proliferación micróglica notable. A veces estos elementos se disponen en discretos nódulos yacentes en la sustancia fundamental. No hay perivascularitis ni formaciones de cuerpos granulosos. Al lado de estos dos elementos lesionales queda un tercero que es la relativa abundancia de vasos, sobrepasando ciertamente los límites de lo normal. Señalamos finalmente la ausencia de lesiones neuroganglionares caracterizables.

Ante las dudas diagnósticas que el caso presentaba practicamos varios bloques en los segmentos superiores del neuroeje y sustancia cerebral. Inmediatamente por debajo del cuerpo calloso, entre éste y el núcleo caudado, existe un foco de desmielinización que contacta con la pared del ventrículo lateral. Este foco tiene el tamaño de un grano de mijo y a simple vista, antes de cortarlo, se aprecia la coloración grisácea del mismo contrastando con la sustancia blanca que lo rodea. Mediante diversas técnicas convergentes se puede poner de manifiesto la existencia de una verdadera placa de desintegración mielinica. En el centro de la misma se ven flotando restos de cilindroejes y algunas células redondas. Los vasos están rodeados de un discreto manguito de cuerpos granulosos gliógenos, de células redondas más pequeñas $\mathrm{y}$, junto con estos elementos, una condensación fibrosa glial perivascular. Por fuera, rodeando como un sacabocados a este centro degenerado, existe una espesa corona de microgliócitos en 

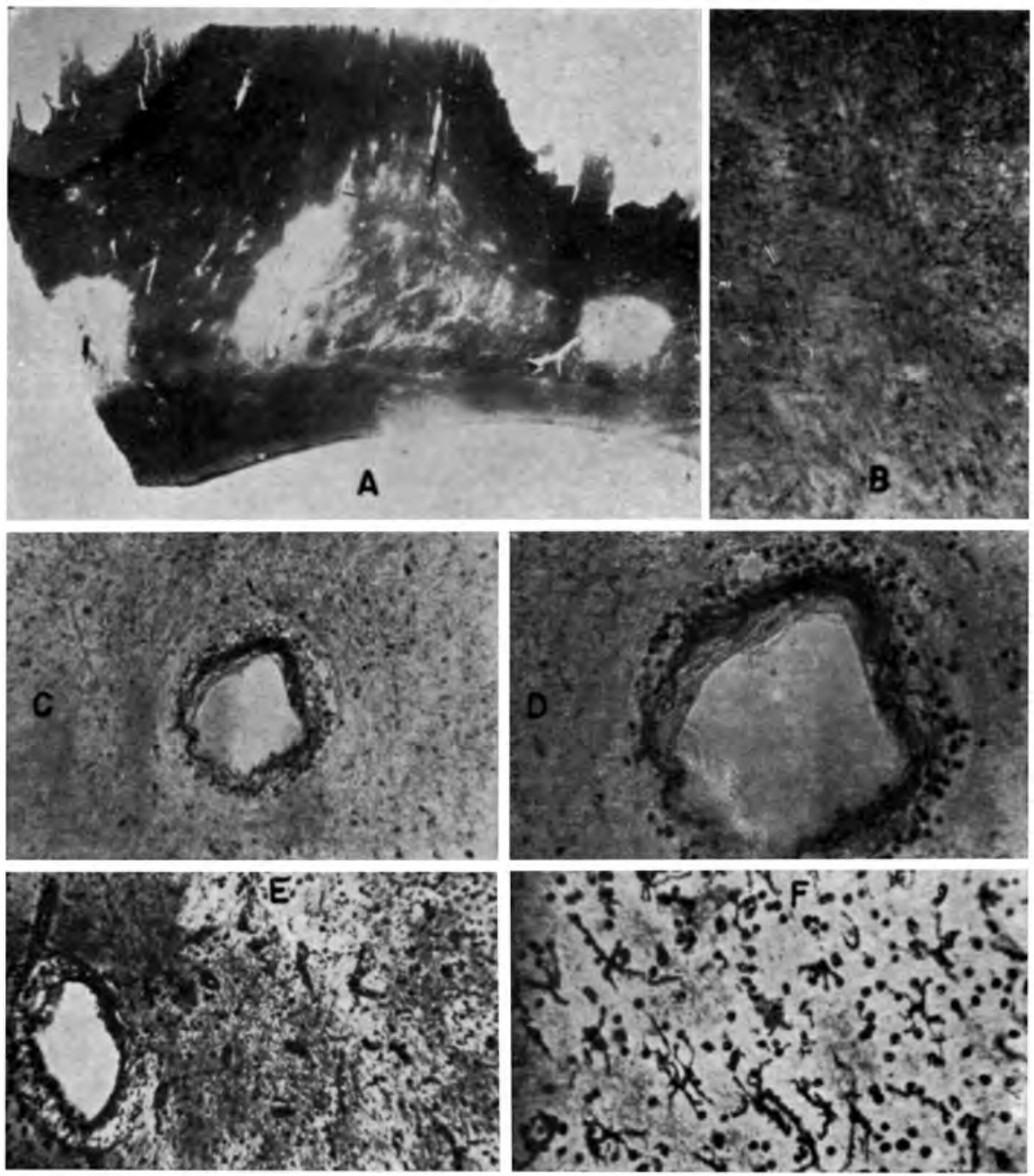

Fig. 1 - Caso A. C. M. En A, placas de desmielinización a disposición perivintricular, situadas en el cuerno occipital (método de Spielmeyer; microplanar). En 13, borde de una placa de esclerosis; obsérvese la transición entre la zona mielinizada normal y la zona desmielinizada (método de Spielmeyer; 200 d.). En C, centro de una placa de esclerosis; obsérvese el vaso rodeado de una corona de cuerpos granulosos (método de Spielmeyer; 200 d.). En D, el mismo vaso que en $C$, visto a mayor aumento. En $E$, proliferación micróglica alrededor de la misma placa vista en las figuras anteriores (método con plata etil-aminada; 100 d.). En F, la misma corona de microgliócitos vista con mayor detalle (plata etil-aminada; 200 d.). 
todos los estadios de actividad. En medio de ellos se observan numerosos vasitos trombosados que rodean la susodicha placa. A nivel de la sustancia periventricular del cuerno occipital, junto al tercer ventrículo, en el tálamo óptico, etc., se registran otras placas que poseen exactamente las mismas características histológicas. Algunas son redondeadas y de tamaño discreto, otras se alargan besando siempre la parede ventricular y resultan de tamaño mucho más considerable. Todas muestran un centro desprovisto de mielina, una corona hecha de astrócitos y microgliócitos y algunos vasos rodeados de discretas perivascularitis.

El tipo de reparación puramente glial, la limpieza de los contornos de la lesión, la desaparición en muchas de las placas de todo resto cilindroaxil, así como la ausencia de una desintegración grasa franca, con formación de cuerpos granulosos, nos inclina a pensar que el proceso es muy antiguo, que ha evolucionado lentamente, pero que no estaba completamente desprovisto de posibilidades evolutivas. Creemos que este cuadro histopatológico entra cómodamente en la más clásica concepción de la esclerosis múltiple. Las microfotografías que acompañan (fig. 1) ilustran algunos de los aspectos señalados.

El interés de la presente contribución casuística anatomoclínica estriba en: a) El contraste entre el cuadro clínico evocador de una afección medular estrictamente localizada en la médula cervical inferior y el diagnóstico histopatológico de un proceso desmielinizante diseminado, esclero. sis múltiple; b) La evolución lentamente progresiva en contradicción con la regla clásica, aunque, como es sabido, no constante; c) La singular mudez clínica de las lesiones cerebrales; d) La enseñanza de que no solo es posible equivocarse tomando por un proceso intrínseco medular (esclerosis múltiple, degeneración combinada subaguda, etc.) una compresión medular cervical (Kahn, Meyer, Fine, Gispert, Obrador y colaboradores, Temple Fay, etc.), sino que también puede cometerse, aunque seguramente ello ocurrirá con menor frecuencia, el error contrario. Este error, como en el caso presente, puede asimismo conducir a consecuencias funestas para el paciente. Ante la duda realmente fundamentada, sin embargo, será en principio preferible ir en busca de la posible compresión; c) La confirmación, por tanto, de que la esclerosis múltiple es en cierto modo la "afección de los contrastes", pudiéndose añadir a tres incongruencias que Wartenberg señala en su aportación antes citada, la frecuente disociación clínico-anatómica entre la relativa concentración topográfica del proceso que hacen suponer los síntomas y signos y la amplia diseminación de las lesiones.

En gracia a la brevedad, virtud a la que todos debiéramos rendir mayor tributo, dejamos por hilvanar numerosos comentarios marginales que nuestra presente observación podría sugerir. De otro modo dejaríamos de atenernos a las normas de concisión, precisión y aportación positiva escueta, que uno de nosotros ha tenido larga ocasión de ir apren- 
diendo al lado del Prof. L. Barraquer Ferré, a cuyo homenaje se acoge este trabajo.

\section{BIBLIOGRAFIA}

1. Barraquer-Bordas, L. - a) Esclerosis Múltiple. Conferencia en el Colégio de Médicos y Hermandad de San Cosme y San Damián de Tarragona, 1 abril 1950; b) Orientaciones diagnósticas ante el sintoma "parestesias en las manos". Med. Clín., 14:360-373, 1950.

2. Barraquer-Bordas, L. y Vila-Badó, J. - Algunas nociones prácticas para el diagnóstico clínico de la esclerosis múltiple. An. de Med. y Cir., 29:62-70, 1951.

3. Schumacher, G. A. - Múltiple sclerosis and its treatment. J.A.M.A., 143: 1059-1065, 1146-1154 y 1241-1250, 1950.

4. Wartenberg, R. - Multiple sclerosis. Post-Grad. M. J., 9:33-34, 1951:

Lauria 102 - Barcelona, España. 